\title{
Direct photolithography on molecular crystals for high performance organic optoelectronic devices
}

\author{
Yifan Yao, ${ }^{\dagger}$ Lei Zhang, ${ }^{*}, \dagger \S$ Tim Leydecker, ${ }^{\dagger}$ Paolo Samorì ${ }^{*}, \dagger$ \\ $\uparrow$ University of Strasbourg, CNRS, ISIS UMR 7006, 8 allée Gaspard Monge, F-67000 Strasbourg, France.
}

\begin{abstract}
Organic crystals are generated via the bottom-up self-assembly of molecular building blocks which are held together through weak non-covalent interactions. Although they revealed extraordinary charge transport characteristics, their labile nature represents a major drawback towards their integration in opto-electronic devices when the use sophisticated patterning techniques is required. Here we have devised a radically new method to enable the use of photolithography directly on molecular crystals, with a spatial resolution below $300 \mathrm{~nm}$, thereby allowing the precise wiring-up of multiple crystals on demand. Two archetypal organic crystals, i.e. p-type diphenyl[1]benzothieno[3,2-b][1]benzothiophene (Dph-BTBT) nanoflakes and n-type N,N'-dioctyl-3,4,9,10perylenedicarboximide (PTCDI-C8) nanowires, have been exploited as active materials to realize high-performance top-contact organic field-effect transistors (OFETs), inverter and p-n heterojunction photovoltaic devices supported on plastic substrate. The compatibility of our direct photolithography technique with organic molecular crystals is key for exploiting the full potential of organic electronics for sophisticated large-area devices and logic circuitries, thus paving the way towards novel applications in plastic (opto)electronics.
\end{abstract}

\section{INTRODUCTION}

Photolithography is nowadays a widely exploited technology which has laid the foundation of modern opto-electronics. Such patterning method has been developed specifically for being used with silicon single crystals to achieve high-level device integration. Due to its versatility, it became a viable strategy towards the engineering of complex and multifunctional micro/nano-scale electronic devices also incorporating two-dimensional (2D) single crystals including graphene, transition metal dichalcogenides ${ }^{2}$ and black phosphorus. ${ }^{3}$ During the last two decades, their non-covalent counterparts, i.e., molecular crystals, have also attracted ever increasing attention as semiconducting building blocks for diverse optoelectronic applications, by taking full advantage of their properties which can be finely tuned through the careful design of the molecular building blocks and their controlled selfassembly. ${ }^{4}$ For example, the formation of 1D and 2D supramolecular assemblies with optimal $\pi-\pi$ stacking enabled the generation of semiconducting architectures for long-distance and extremely efficient charge transport yielding high charge carrier mobility devices which can be also supported on flexible substrates. ${ }^{5}$ Since the self-assembly of molecular building blocks typically leads to the formation of ordered structures in the form of discrete and anisotropic organic crystals instead of uniform thin-films, ad-hoc micro-/nano-fabrication methods based on the use of e-beam lithography (EBL) or focused-ion beam (FIB) are typically required to precisely control their wiring-up with metallic electrodes. Unfortunately, these sophisticated patterning technologies are invasive, as they rely on high energy beams, thus cannot be employed directly on molecular crystals. ${ }^{6}$ To date, only very few methods to fabricate devices incorporating such "small" organic crystals have been devised. $\mathrm{Hu}$ and co-workers have proposed some approaches for the in-situ and non-destructive wiring-up of "small" crystal into nanodevices which relied on the stamping of electrodes and the use of organic ribbons as mask. ${ }^{7}$ These manual methods could enable the integration of an individual micro-/nano-crystal in order to test its intrinsic (opto)electronic properties, but they are unsuitable for the processing of complex multicomponent structures and batch production towards commercial products.

In light of this, photolithography still remains the ideal method to integrate molecular crystals into (opto)electronic devices. The possibility of extending photolithography to organic crystals would enable to fully exploit their intrinsic properties by precisely patterning electrodes on their top to finally merge inorganic and organic materials towards highperformance hybrid optoelectronic solutions. However, such a task is extremely challenging because of the intrinsic fragility and instability of molecular crystals when exposed to the solvents needed to process the photoresist and to the alkaline developer. Furthermore, molecular crystals are too brittle to undergo through the photoresist lift-off process.

Here we report a novel procedure enabling the use photolithography to directly integrate multiple organic crystals simultaneously into micro-/nano optoelectronic devices and electrodes could be patterned with an arbitrary shape and position with a high degree of precision. The interelectrodic distance could be downscaled to ca. $300 \mathrm{~nm}$. By taking advantage of the short-channel geometry, ultrasensitive crystalline phototransistors with a photoresponsivity up to $4.8 \times 10^{4} \mathrm{~A} / \mathrm{W}$ under $500 \mathrm{~nm}$ wavelength were realized. Significantly, this methodology is compatible with flexible substrates such as polyeth- 
ylene terephthalate (PET). In this regard, as a proof-of-concept we have

(a)

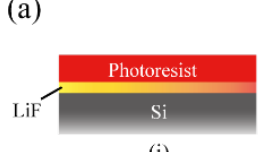

(i)
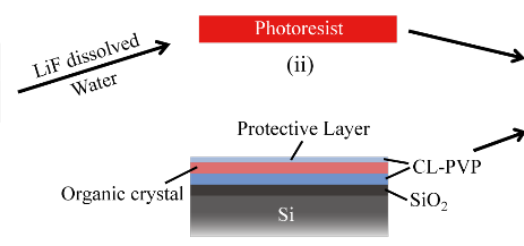

(iii)

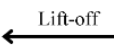

(viii)

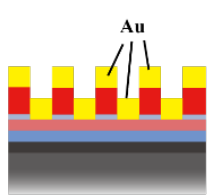

(vii)

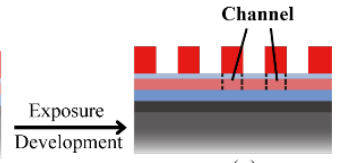

(v)

(iv)

$\stackrel{\text { Metal deposition }}{\longleftarrow}$

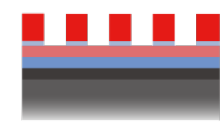

(vi)
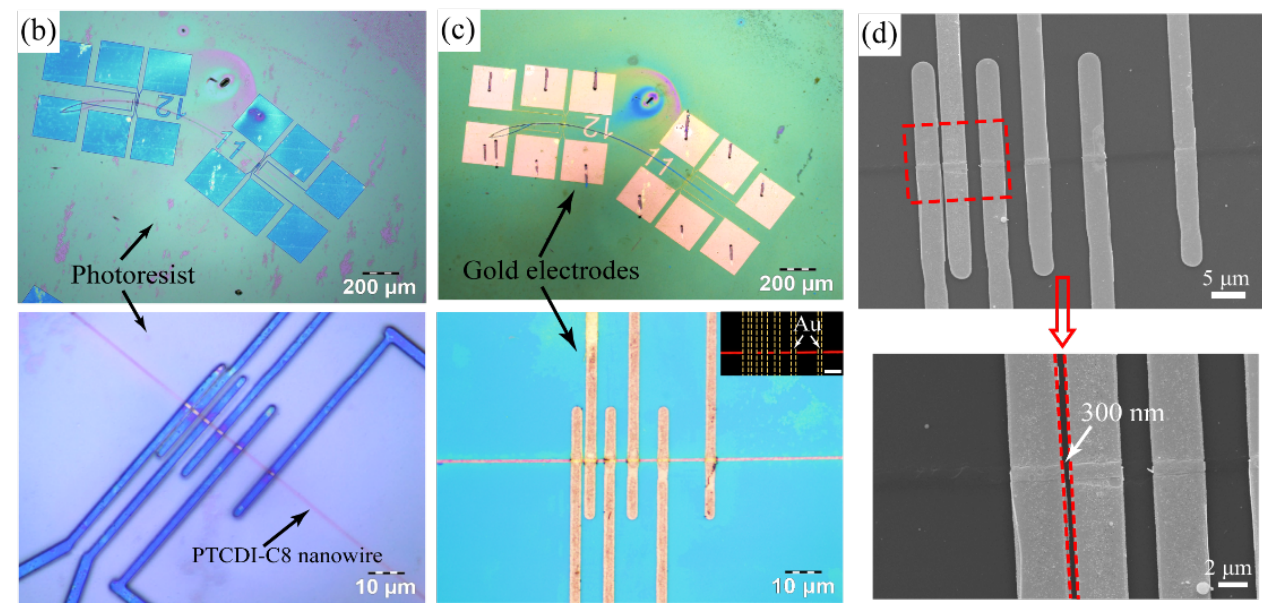

Figure 1. (a) Schematic diagram of the direct photolithography technique. (i) A layer of photoresist (thickness ca. $1 \mu \mathrm{m}$ ) is spincoated on a hydrophilic Si substrate which has been previously covered with a sacrificial $\sim 110 \mathrm{~nm}$ thick layer of LiF. (ii) In order to peel-off the photoresist layer from the substrate, water is used to dissolve the LiF sacrificial layer. As a result, the photoresist film then floats on the water surface. (iii) CL-PVP is used to modify the $\mathrm{SiO}_{2} / \mathrm{Si}$ substrate and to encapsulate the organic semiconductor layer. (iv) The photoresist film is then transferred onto the sample substrate bearing the encapsulated molecular crystals. (v) Exposure and development are used to generate the desired pattern on the photoresist film. (vi) Oxygen plasma enabled to etch away the protective layer on OSC layer. (vii) Metal deposition and (viii) mild lift-off process in warm ethanol. (b) Optical microscopy images of a single PTCDI-C8 nanowire after exposure, development and etching. (c) Optical microscopy images of PTCDI-C8 based FET device after metal deposition and lift-off. Inset shows the fluorescence microscopy image of PTCDI-C 8 crystal (the scale bar denotes $10 \mu \mathrm{m}$ ). (d) SEM image of patterned electrodes. Bottom inset zoom-in SEM image of the same device in (d), the narrowest channel is around $300 \mathrm{~nm}$.

fabricated organic field-effect transistors (OFETs) and complementary inverter circuits, based on p-type diphenyl[1]benzothieno[3,2-b][1]benzothiophene (Dph-BTBT) nanoflakes and n-type $\mathrm{N}, \mathrm{N}^{\prime}$-dioctyl-3,4,9,10perylenedicarboximide (PTCDI-C8) nanowires, supported on PET foils. The work function of the electrodes could also be tuned by choosing different metals like gold and silver yielding asymmetric contacts with the chosen molecular crystals. Furthermore, organic crystalline donor-acceptor (D-A) heterojunction photovoltaic devices based on planar asymmetric AuAg electrode pairs have also been developed. The compatibility and versatility of our photolithography technique with organic molecular crystals is key towards the development of sophisticated large-area devices, thus paving the way towards advanced opto-electronic applications.

\section{RESULTS AND DISCUSSION}

To circumvent any problem related to the exposure of the fragile organic crystal to the standard conditions of photolithography processes, here we have devised a novel procedure
(Figure 1a), which starts with the deposition of the photoresist on the top of a water-soluble interlayer. In particular, a centimeter-sized photoresist film (thickness ca. $1 \mu \mathrm{m}$ ) was peeled off from the hydrophilic Si wafer by exploiting a $100 \mathrm{~nm}$ thick lithium fluoride $(\mathrm{LiF})$ sacrificial layer and by using the waterfloatation method. Figure S1 shows a photograph of such freestanding photoresist film being removed from a silicon substrate and subsequently transferred onto a crosslinked poly(4vinylphenol) (CL-PVP) modified substrate. In order to protect the organic crystals from contamination during the development of the photoresist, we optimized the traditional photolithography procedure by encapsulating the crystals through spin-coating an additional $45-50 \mathrm{~nm}$ thick CL-PVP protective top layer. The CL-PVP layer not only protected the organic semiconducting crystals (OSCs) against the alkaline developer solution but also immobilized the micro/nano-crystals during the lift-off process. The floating and freestanding photoresist film (Figure 1a ii) was transferred onto such a CL-PVP protected target substrate. Photo exposure using a laser-writer operating with a $405 \mathrm{~nm}$ laser followed by conventional de- 
velopment process enabled direct patterning onto the crystals. Prior to the evaporation of a gold layer, oxygen plasma was used in order to carefully remove the CL-PVP protecting layer thereby ensuring the generation of intimate electrical contact between the OSCs and gold electrodes. Noteworthy, the removal and lift-off process of the photoresist was carried out using warm ethanol which is a non-solvent for most organic semiconducting materials. An array of patterned electrodes on large area substrate is displayed in Figure S1c-e. Scanning electron microscope (SEM) images show that the shortest channel length (L) can be patterned is ca. $250 \mathrm{~nm}$ long. The leakage current passing through such a junction is on the 1-to$10 \mathrm{pA}$ scale, thus approaching the noise limit of our electrical measurement set-up. Such estimation confirms the absence of residual gold leftovers within the channel and therefore provides evidence for the success of the lift-off process carried out in ethanol (see Figure S1f-g).

In order to show that our novel direct photolithography procedure can be applied to devices integrating active organic molecular crystals, highly aligned n-type PTCDI-C8 nanowires self-assembled via the "capillary tube" method and ptype Dph-BTBT nanoflakes grown through "solvent-induced precipitation (SIP)" have been tested as prototypical nanostructures. ${ }^{8}$ The growth of laterally stacked PTCDI-C8 nanowires on surfaces is schematically depicted in Figure S2a. In order to form aligned crystals over a large-area, the PTCDIC8 solution in 1,2-dichlorobenzene was deposited on a needle and allowed to crystallize at $65{ }^{\circ} \mathrm{C}$ on a hot plate for $1 \mathrm{~h}$. The obtained nanowires exhibited their long axis oriented perpendicular to the needle. Figure S2b-d shows the optical microscopy and fluorescence images of the PTCDI-C8 crystals. The optical image of the PTCDI-C8 crystals covered with the CLPVP (Figure S2e) provides evidence that all the crystals remained intact when subjected to the encapsulation process.

Figure $1 \mathrm{~b}$ portrays the optical microscope images of a single PTCDI-C8 nanowire embedded in the patterned photoresist layer after exposure, development and $\mathrm{O}_{2}$ plasma etching (stage displayed in Figure 1a vi). Metal electrodes with different channel lengths could be micro-fabricated onto the crystal. The device obtained after metal deposition and lift-off (stage displayed in Figure 1a viii) are shown in Figure 1c. Fluorescence microscopy images of the PTCDI-C8 crystal recorded after the previously discussed processing steps confirms the full crystal integrity (inset of Figure 1c). The SEM images of the patterned electrodes in Figure 1d demonstrate that in our top electrodes configuration the channel length in devices integrating the PTCDI-C8 crystal can be as small as $\sim 300 \mathrm{~nm}$, being one among the best results for top-contact configuration OFETs. 6

The effect of oxygen plasma treatment on the morphology and electrical performance of PTCDI-C8 nanowires have been thoroughly investigated by tuning the etching dose. The results are summarized in the Figure S3-S6 and Table S1. In the case of PTCDI-C8 nanowires encapsulated by CL-PVP, the morphology has been characterized at different oxygen etching levels by AFM (Figure S4). The increasing etching duration is accompanied by a gradual increase in the crystal surface roughness, yet the crystal shape remains unaltered. This observation indicates that the oxygen plasma only affects the surface of the crystals whereas the crystal structure remains largely preserved. Importantly, the charge carriers transport channel is well protected by the photoresist (Figure 1a v) and are not influenced by

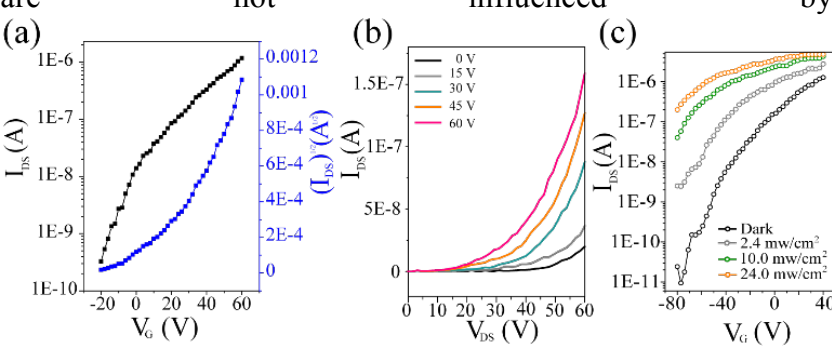

(d)

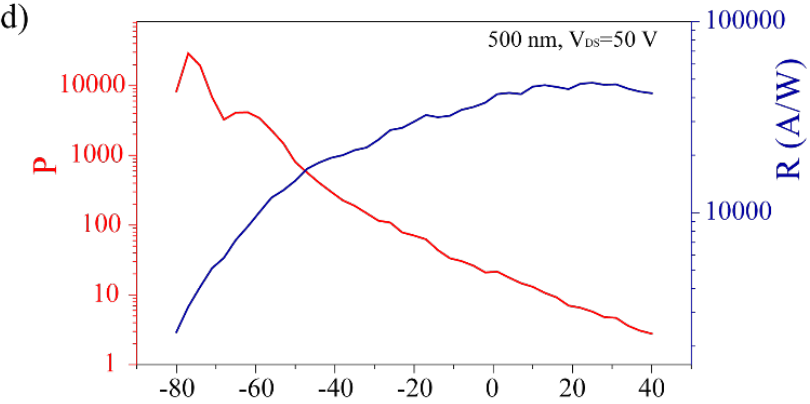

(e)

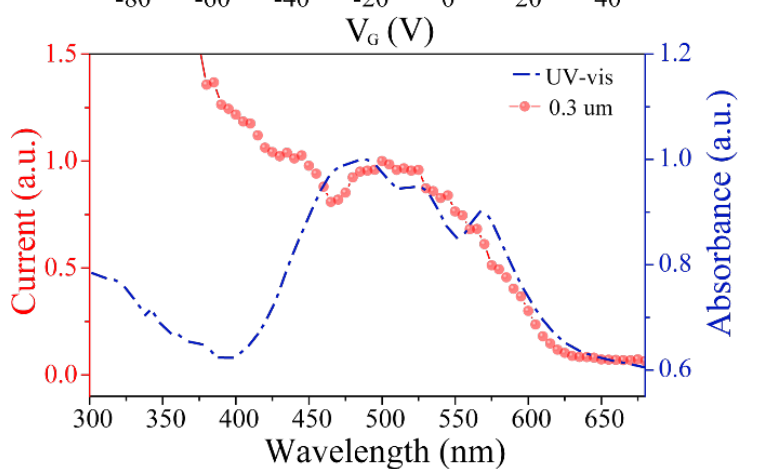

Figure 2. Optoelectronic response of a PTCDI-C8 nanowire short channel transistors. Transfer (a) and output (b) characteristics of the PTCDI-C8 nanowire transistor. (c) The optoelectronic response of photo-gated OFET based on PTCDI-C8 nanowire. (d) Photoresponsivity (R) and photosensitivity (P). (e) Normalized photocurrent spectrum versus normalized UV absorption. The channel length was $300 \mathrm{~nm}$ for all the above measurements.

the oxygen etching. The transfer and output curves of the resulting device with different oxygen etching duration are displayed as Figure S5, S6 and the statistics are reported in the Table S1. After 85 s oxygen etching duration PTCDI-C8 crystal FET devices exhibited electron mobility of $0.18 \pm 0.09 \mathrm{~cm}^{2}$ $\mathrm{V}^{-1} \mathrm{~s}^{-1}, \mathrm{I}_{\text {on }} / \mathrm{I}_{\text {off }}$ of $6.2 \times 10^{4}$ and threshold voltage of $34 \pm 7 \mathrm{~V}$, which are extremely similar to those previously reported in the literature. ${ }^{8}$ Such indicators confirm that oxygen etching has a minor influence on the electrical performance of the OFETs.

Our novel direct photolithography technique makes it possible to study the short channel effect on the electron mobility and photoresponse on a single PTCDI-C 8 nanowire by making multiple top-contacts in a bottom-gate OFETs configuration. The results are shown in Figure 2a-e and Figure S7-S12. In order to exclude possible effects of contact resistance $\left(\mathrm{R}_{\mathrm{c}}\right)$ or bottlenecks to charge transport due by the presence of grain boundaries on the device performance, we have studied the identical nanowire at different channel lengths in order to cast light onto geometrical parameters which could affect the photoresponse. The contact resistance, as determined by means of 

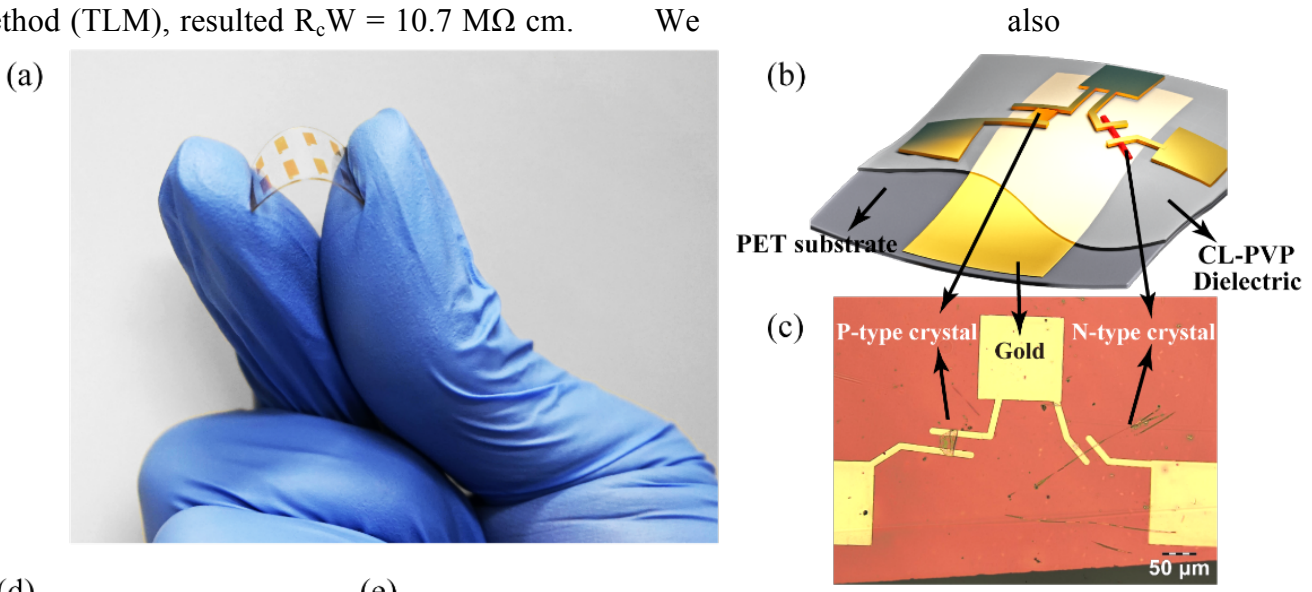

observed

(d)

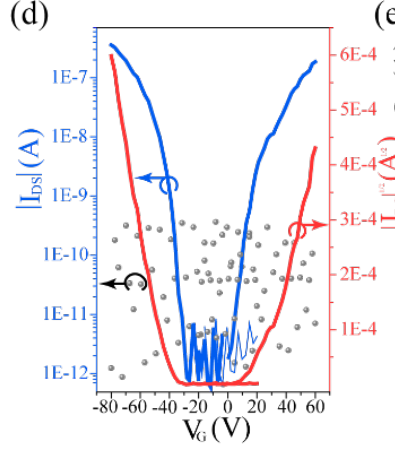

(e)

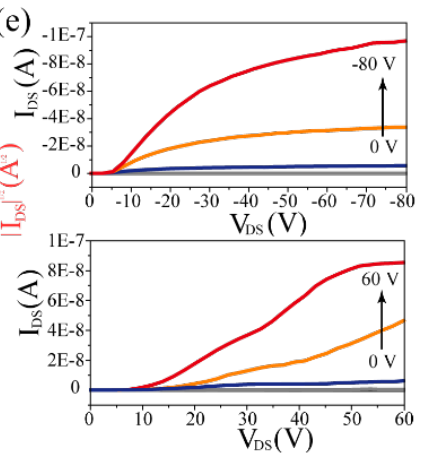

(f)

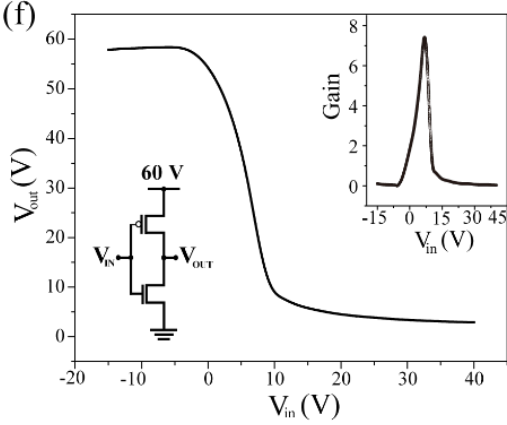

Figure 3. Optical image of CMOS inverters on flexible PET substrate, schematic diagrams of a device and its electrical characterization. (a) Photograph of as-fabricated CMOS inverters based on molecular crystals on flexible PET substrate. (b) 3D schematic and corresponding optical microscopy image of such a CMOS inverter (c). Transfer (d) and output (e) characteristics of individual p-type and n-type OFETs on PET substrate. (f) Output voltage $\left(\mathrm{V}_{\text {out }}\right)$ as a function of input voltage $\left(\mathrm{V}_{\text {in }}\right)$ and the device was driven by $a \quad V_{D D}(60 \quad \mathrm{~V})$. The inset indicates the gain of the inverter. that for $\mathrm{L}$ ranging between 300 and $500 \mathrm{~nm}$, the output charac- $\quad P$ on the channel length as measured on the identical PTCDIteristics exhibit the well-known "S" shaped curves (Figure 2b). The absence of saturation can be ascribed to the "shortchannel effect". ${ }^{10}$ The effect of the channel length on the fieldeffect mobility and resistance of single PTCDI-C 8 nanowire is summarized as Figure S7 and Table S2. In particular, the statistical analysis based on 23 different OFETs integrating an individual PTCDI-C8 nanowire revealed that upon increasing the channel length (L) the device exhibits a higher electron mobility, accompanied by a reduced role played by the contact resistance. ${ }^{11}$ The electron mobility amounted to $\mu_{\mathrm{e}}=0.31 \pm 0.11$ $\mathrm{cm}^{2} \mathrm{~V}^{-1} \mathrm{~s}^{-1}$ for a device with $\mathrm{L}=10 \mu \mathrm{m}$ displaying a $\mathrm{V}_{\text {th }}=$ $34.1 \pm 6.9 \mathrm{~V}$ and $\mathrm{I}_{\mathrm{on}} / \mathrm{I}_{\text {off }}$ ratio $=1.6 \times 10^{5}$. When silver electrodes are patterned instead of gold (Figure S8) an even higher electron mobility of $\mu_{\mathrm{e}}=0.40 \pm 0.35 \mathrm{~cm}^{2} \mathrm{~V}^{-1} \mathrm{~s}^{-1}$ along with $\mathrm{V}_{\text {th }}=$ $37.3 \pm 6.4 \mathrm{~V}$ and $\mathrm{I}_{\mathrm{on}} / \mathrm{I}_{\text {off }}$ ratio $=1.3 \times 10^{4}$ has been measured for the same L. Such an improvement in the device performance proves that Ag could form a better ohmic contact with PTCDI$\mathrm{C} 8$ nanowires compared to $\mathrm{Au}$.

When incorporated as light sensitive component of the phototransistors, the photo responsivity of PTCDI-C8 nanowires could in principle also be affected by the channel length. Transfer curves were recorded under light illumination $(\lambda=$ $500 \mathrm{~nm}$ ) with different intensities and channel lengths. With the increasing intensity, the transfer curves shifted to higher gate voltages with a gradual reduction of $\mathrm{I}_{\mathrm{on}} / \mathrm{I}_{\text {off }}$ ratio, as displayed in the Figure 2c. Figure S9 and Table S3 show the dependence of the photoresponsivity $\mathrm{R}$ and the photosensitivity

$\mathrm{C} 8$ nanowire. The photosensitivity $\mathrm{P}$ is defined as

$$
\mathrm{P}=\frac{I_{\text {light }}-I_{\text {dark }}}{I_{\text {dark }}}
$$

where $I_{\text {light }}$ and $I_{\text {dark }}$ are the drain current under illumination and in dark, respectively. The photoresponsivity $\mathrm{R}$ being

$$
\mathrm{R}=\frac{I_{\text {light }}-I_{\text {dark }}}{S \cdot P_{i}}
$$

With $P_{i}$ is the incident light intensity (usually expressed in $\mathrm{W} / \mathrm{cm}^{2}$ ), and $\mathrm{S}$ is the effective device area.

For $\mathrm{L}=300 \mathrm{~nm}$, displayed in the Figure $2 \mathrm{~d}$, the $\mathrm{R}$ and $\mathrm{P}$ values amount to $4.8 \times 10^{4} \mathrm{~A} / \mathrm{W}$ and $2.9 \times 10^{4}$, respectively. This result demonstrating that the use of short channel geometries can harness the optoelectronic properties of organic semiconducting materials, overall leading to improved device performances, e.g. in phototransistors (OPTs) ${ }^{12}$ and ultra-fast photodetectors. ${ }^{13}$ Here, on the single PTCDI-C8 nanowire, we can draw a solid conclusion that the geometrical reduction of channel length yields devices featuring increased responsivity and photosensitivity. Such findings are supported by the evidence that shorter channel length devices exhibit higher source-drain currents, less traps and less grain boundaries, thus approaching the intrinsic characteristics of perfect organic molecular crystals. ${ }^{14}$

Phototransistors are based on photo-generated free charge carriers, ${ }^{15}$ thus the photocurrent measured in these devices depends on the number of photons absorbed by the active material. The absorption spectrum of PTCDI-C8 is displayed in 
blue dashed in Figure 2e. The photoresponse characteristics of sweeping the devices for different wavelength was investigated by
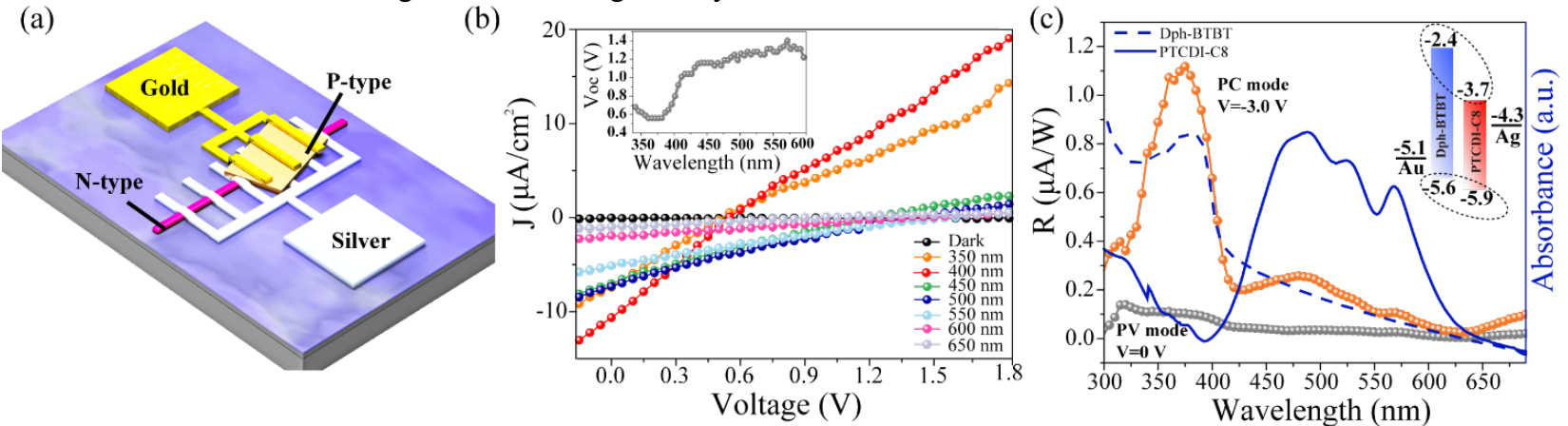

Figure 4. Photovoltaic effect in heterojunction devices based on PTCDI-C8 nanowires and Dph-BTBT nanoflakes. (a) 3D schematic diagram of the D-A heterojunction photovoltaic device based on two different crystals, i.e., Dph-BTBT nanoflakes and PTCDIC8 nanowires. (b) J-V curves in the dark and upon illumination at different wavelength. The inset shows the open-circuit voltage significantly increases from $0.56 \mathrm{~V}$ to $1.4 \mathrm{~V}$ when the illumination wavelength changed from $320 \mathrm{~nm}$ to $690 \mathrm{~nm}$. (c) Photoresponsivity spectra in PC mode $(-3.0 \mathrm{~V}$ bias $)$ and PV mode $(0 \mathrm{~V}$ bias $)$. The blue dashed and solid line indicate the absorption spectra of Dph-BTBT nanoflakes and PTCDI-C8 nanowires, respectively. The inset shows the HOMO-LUMO energy levels of both materials.

the irradiation wavelength from 320 to $690 \mathrm{~nm}$. Since the light power density upon wavelength is also uneven, we divide photocurrents of each channel length by the spectral power density function of our light source (Figure S10) to obtain the normalized photocurrents shown in Figure 2e and Figure S11. We found that the photocurrent is strongly dependent on the channel length. For the narrowest channels, the normalized current trends comply with the absorption spectrum of PTCDI-C8 crystal, because of the low density of defects. The tailing effect appears at $\lambda>470 \mathrm{~nm}$ due to the slow response to light. ${ }^{7}$ Figure S12 portrays the transient behavior of the device under illumination. The $\mathrm{V}_{\mathrm{DS}}$ was kept constant at $40 \mathrm{~V}$ while the gate voltage, light wavelength and intensity were set constant values of $-70 \mathrm{~V}, 500 \mathrm{~nm}$ and $24 \mathrm{~mW} / \mathrm{cm}^{2}$, respectively. By switching the light source on and off, the devices were capable of being switched on/off reversibly with high reproducibility and no fatigue. However, the response speed of the photocurrent was found to be independent on the channel length. Such evidence means that photon-generated carrier recombination and transfer rate are dominant, due to a slow release of accumulated and trapped electrons in the semiconductor-gate dielectric interface, rather than the channel length. ${ }^{16}$

The high resolution direct photolithography technique can be readily scaled up to provide a high efficiency and reliable tool to construct organic semiconductor-based integrated circuits. Complementary metal-oxide-semiconductor (CMOS) as the basic unit in logic circuits exhibit the advantages of high signal amplification gain, low power dissipation and high robustness. In a CMOS inverter p-type and n-type semiconductors operate simultaneously. In order to demonstrate complementary logic circuit based on molecular crystals, we have prepared a large number of p-type Dph-BTBT (Figure S13) nanoflakes via solvent induced precipitation (SIP). Nanoflakes with different shapes can be obtained as shown in Figure S14. We have focused our attention on nanoflakes grown in chlorobenzene/ethanol because they featured the most regular shapes. Those p-type nanoflakes were then transferred onto the substrate previously coated with drop-casted PTCDI-C8 nanowires (Figure S15). Finally, CMOS inverters were realized by wiring-up two unipolar crystal FET devices based on DphBTBT nanoflakes and PTCDI-C8 nanowires through direct photolithography (Figure S16). The performance of individual OFETs constituting CMOS inverter on $\mathrm{SiO}_{2}$ substrate is shown in Figure S17. According to the transfer characteristics, the field-effect electron mobility of the n-type PTCDI-C8 nanowire device results $0.13 \mathrm{~cm}^{2} \mathrm{~V}^{-1} \mathrm{~s}^{-1}$. Moreover, individual $\mathrm{p}$ type Dph-BTBT nanoflake devices also exhibit an unambiguous FET character, with hole mobilities reaching $0.1 \mathrm{~cm}^{2} \mathrm{~V}^{-1} \mathrm{~s}^{-}$ 1. Such results testify the high quality of Dph-BTBT nanoflakes and PTCDI-C8 nanowires as reliable individual OFET. The static responses of the CMOS-inverter based on the p-type and n-type crystals OFETs have been analyzed. The voltage transfer characteristics of the inverter are depicted in Figure S17d. They exhibit good inversion characteristics in $\mathrm{V}_{\text {out }}$ with a gain as high as $\sim 11$, with an excellent noise margin of $49 \%$ of $1 / 2$ VDD.

We have also applied this photolithography technique to fabricate crystals based CMOS-inverters on flexible substrate such as PET (Figure 3a). In this case, a $600 \mathrm{~nm}$ thick CL-PVP film (Figure S18) was used as dielectric layer coating the prepatterned gold gate electrodes. N-type and p-type crystals were grown and transferred onto the substrate by using the same methods as described above. The schematic structure and photograph of the crystal-based CMOS inverters are shown in Figure $3 b-c$. The transfer and output curves of FETs incorporated in the CMOS-inverter exhibit well-balanced charge transport behavior and a very low gate leakage current as displayed in Figure 3d-e. The FETs mobility in the inverter configuration amounted to $\mu_{\mathrm{e}}=0.16 \mathrm{~cm}^{2} \mathrm{~V}^{-1} \mathrm{~s}^{-1}$ and $\mu_{\mathrm{h}}=0.26$ $\mathrm{cm}^{2} \mathrm{~V}^{-1} \mathrm{~s}^{-1}$ with $\mathrm{I}_{\text {on }} / \mathrm{I}_{\text {off }}$ ratios of $6.1 \times 10^{5}$ and $1.3 \times 10^{4}$, respectively. The complementary inverter showed the typical switching characteristic with a gain of $\sim 7$ (Figure $3 \mathrm{f}$ ), being slightly lower than the one observed on $\mathrm{Si} / \mathrm{SiO}_{2}$ substrate due to the difference in capacitance between the $230 \mathrm{~nm}$ thick $\mathrm{SiO}_{2}$ surface and the $600 \mathrm{~nm}$ thick CL-PVP film. The ability to construct CMOS inverters on micro-scale organic crystals by using the reliable and high-resolution approach is highly encouraging. It should be emphasized that the fabrication method developed and device structure optimization made in this work are not limited to specific molecular active systems, as it is compatible with large quantity of organic and polymeric conjugated materials, and can be readily used to construct large- 
scale logic circuits with a better performance for future flexible electronic applications.

Organic p-n crystalline heterojunctions possess the advantages of long exciton diffusion and fast charge transport which are of paramount importance for excitons dissociation and photocurrent generation in photovoltaics. ${ }^{17} \mathrm{Li}$ et al. demonstrated that extended single-crystalline heterojunctions obtained by simple solution processing can possess a power conversion efficiency (PCE) of $0.46 \%{ }^{17 \mathrm{c}}$ Significantly, the direct photolithography approach we have developed here also allows to micro-fabricate different metals onto crystalline organic heterojunction with desired patterns by repeating the photolithography in sequence. In this way, it is possible to realize planar organic photovoltaic devices based on DphBTBT nanoflake and PTCDI-C8 nanowire which are selectively connected to an asymmetric Ag-Au interdigitated electrodes pair. The device fabrication procedure is illustrated in Figure S19. It is worth noting that the ultra-thin $(10-20 \mathrm{~nm}$ thick) Dph-BTBT nanoflakes are conformal enough to generate an intimate contact with both PTCDI-C8 nanowires and the silver electrode, which is the prerequisite for the photogenerated excitons to effectively dissociate at the p-n heterojunction interface and collect by the electrodes.

Two control experiments have been carried out by fabricating devices integrating only a single PTCDI-C8 nanowire, in absence of the p-type component. When the symmetrical gold electrodes are used to connect the PTCDI-C8 nanowire, no photovoltaic effect was observed (Figure S20a,b). If one of the gold electrode was replaced by a silver electrode to form an asymmetric Ag-Au electrodes pair, unambiguous photovoltaic effect appeared thanks to the formation of asymmetrical Schottky barriers and a built-in electric field. However, the key parameters such as open-circuit voltage $\left(\mathrm{V}_{\mathrm{oc}}\right)$ and shortcircuit current $\left(\mathrm{J}_{\mathrm{sc}}\right)$ are quite low, amounting only to $80 \mathrm{mV}$ and $0.2 \mu \mathrm{A} / \mathrm{cm}^{2}$, respectively (Figure $\mathrm{S} 20 \mathrm{c}, \mathrm{d}$ ). ${ }^{18}$ In order to further enhance the photovoltaic efficiency, here we have introduced p-type crystals to form crystalline heterojunction with n-type PTCDI-C8 nanowire and serve as exciton dissociation interface. The device schematic diagram is shown in Figure $4 \mathrm{a}$, and the survey optical image of the device is displayed in Figure S20e. The photovoltaic effect could be largely enhanced due to the formation of high quality $p-n$ interface. For example, the $\mathrm{J}_{\mathrm{sc}}$ has increased from 0.2 to $7.3 \mu \mathrm{A} / \mathrm{cm}^{2}$ under $500 \mathrm{~nm}$ wavelength, which is 37 times larger than the result in unipolar PTCDI-C8 nanowire with asymmetric Ag-Au electrodes. The current density $(\mathrm{J})$ vs. voltage applied at irradiation with different wavelength are plotted in Figure $4 \mathrm{~b}$, together with the dark current. The light power density under different wavelength is displayed in Figure S10f. According to the UV-vis absorption spectra when the irradiation light wavelength ranges $350 \mathrm{~nm}$ and $400 \mathrm{~nm}$, only p-type Dph-BTBT nanoflakes absorb photons (blue dashed line in Figure 4c), thus contribute to the photovoltaic effect. In this case, the $\mathrm{V}_{\mathrm{oc}}$ is located at about $0.5-0.6 \mathrm{~V}$. However, for the wavelength of 450-600 nm, PTCDI-C8 crystal (blue solid line shown in Figure $4 \mathrm{c}$ ) is more sensitive than Dph-BTBT. The excitons are indeed mainly generated in the PTCDI-C8 crystals and the $\mathrm{V}_{\mathrm{oc}}$ dramatically increases to $\sim 1.3 \mathrm{~V}$. In Figure $4 \mathrm{c}$, we have investigated the photoresponsivity of the crystalline heterojunction devices in photovoltaic (PV) and photoconduction (PC) modes at different wavelength. The photocurrent is apparently deter- mined by the absorption in PTCDI-C8 nanowire and DphBTBT nanoflake. By applying a working voltage, the photoresponsivity could be largely enhanced due to the photoconduction effect. For example, the responsivity has increased from 0.1 to $1.1 \mu \mathrm{A} / \mathrm{W}$ by applying a $-3.0 \mathrm{~V}$ bias voltage. The magnified image at $\lambda=420-690 \mathrm{~nm}$ is shown in Figure S20f.

Such a significant $\mathrm{V}_{\text {oc }}$ spectrum regulation is due to the energy level alignment between Dph-BTBT and PTCDI-C8, which was drawn as the inset of Figure 4c. Here we should note the main driving force for exciton separation at the donor/acceptor interface are $\triangle \mathrm{HOMO}$ and $\triangle \mathrm{LUMO}$ respectively. ${ }^{19}$ In other words, the energy dissipation during the exciton dissociation process has been linearly determined by the $\triangle \mathrm{HOMO}$ and $\triangle \mathrm{LUMO}$. As for Dph-BTBT/PTCDI-C8 donoracceptor pair, $\triangle \mathrm{LUMO}$ is much larger than $\triangle \mathrm{HOMO}(1.3 \mathrm{eV}$ vs. $0.3 \mathrm{eV})$ in the heterojunction. For example, when the wavelength of the light is located at $\lambda=350-400 \mathrm{~nm}$, the excitons are mainly generated in the Dph-BTBT nanoflake; the large $\triangle$ LUMO energy level mismatch $(1.3 \mathrm{eV})$ leads to more efficient electron separation but greater energy loss during the electrons transfer from Dph-BTBT to neighboring PTCDI-C8 crystal. In contrast, when excitons are generated in PTCDI-C8 crystal $(\lambda=450 \mathrm{~nm}-600 \mathrm{~nm})$ and get separated at the heterojunction interface, less energy is dissipated thanks to the much smaller $\triangle \mathrm{HOMO}$ energy level difference (only $0.3 \mathrm{eV}$ ). In line with our observation, such a different energy loss by irradiating different components would tune the $\mathrm{V}_{\mathrm{oc}}$ to a certain extent. ${ }^{13}$ Furthermore, there is a competition between $\mathrm{V}_{\mathrm{oc}}$ and $\mathrm{J}_{\mathrm{sc}}$ : a larger $\triangle \mathrm{LUMO}(1.3 \mathrm{eV})$ than the $\triangle \mathrm{HOMO}(0.3 \mathrm{eV})$ means stronger driving force for the excitons in Dph-BTBT crystal to get separated. As a result, the short circuit photocurrent originated from Dph-BTBT is also greater than that from PTCDIC8.

\section{CONCLUSION}

We have demonstrated a radically new, non-invasive and universal photolithography based method which can be exploited for the micro/nano-fabrication of opto-electronic devices incorporating fragile organic crystals. The versatility of our fabrication strategy allows to modulate the channel length of the devices in the range of hundreds nanometer up to tens or even hundreds micrometer and explore its effect on the fieldeffect mobility and light response of single PTCDI-C8 nanowire integrated in a top-contact bottom-gate configuration. The organic crystal based FETs fabricated by direct photolithography also exhibited a high yield and performance uniformity. Importantly, this photolithography technique is also compatible with flexible substrates, as demonstrated through the fabrication of micro/nano organic crystals based CMOS inverters on PET substrate. Furthermore, the possibility to copattern electrodes with different metals enables the realization of organic crystalline donor-acceptor (D-A) heterojunction photovoltaic devices based on planar asymmetric Au-Ag electrodes. Interestingly, we have found an adjustable drift of open-circuit voltage $\left(\mathrm{V}_{\text {oc }}\right)$ from $0.56 \mathrm{~V}$ to $1.4 \mathrm{~V}$ when the irradiation wavelength being tuned from $320 \mathrm{~nm}$ to $690 \mathrm{~nm}$. Our strategy, relying on the use of direct photolithography on high quality molecular crystals, represents a major step forward being an efficient, reliable and robust approach towards the fabrication of large-area and flexible organic nano-electronics and facilitates the application of sophisticated lithography for 
plastic-electronics. In the future, new proof-of-concept devices based on organic-inorganic hybrids could also be conceived taking full advantage of the fact that photolithography technology has become compatible with both components.

\section{ASSOCIATED CONTENT}

Supporting Information: The Supporting Information is available free of charge on the ACS Publications website at DOI.

Experimental methods, photolithography process, preparation of PTDCI-C8 nanowires and Dph-BTBT nanoflakes, AFM images, statistical mobilities and optoelectronic measurements are included in the supporting information.

\section{AUTHOR INFORMATION}

\section{Corresponding Author}

*samori@unistra.fr

* 1ei.zhang@unistra.fr

\section{Present Addresses}

$\S$ Key Laboratory of Mesoscopic Chemistry of MOE, School of Chemistry and Chemical Engineering, Nanjing University, Nanjing, Jiangsu 210023, China

\section{Notes}

The authors declare no competing financial interests.

\section{ACKNOWLEDGMENT}

This work was financially supported by EC through the ERC Proof-of-Concept project FlexNanoOLED (GA-766936) and the Marie Curie ITN project iSwitch (GA No. 642196), the Labex project CSC (ANR-10-LABX-0026 CSC) within the Investissement d'Avenir program ANR-10-IDEX-0002-02, and the International Center for Frontier Research in Chemistry (icFRC).

\section{REFERENCES}

(1) (a) Geim, A. K., Science 2009, 324, 1530-1534. (b) Geim, A. K.; Novoselov, K. S., Nat. Mater. 2007, 6, 183-191.

(2) (a) Wang, Q. H.; Kalantar-Zadeh, K.; Kis, A.; Coleman, J. N.; Strano, M. S., Nat. Nanotechnol. 2012, 7, 699-712. (b) Novoselov, K. S.; Jiang, D.; Schedin, F.; Booth, T. J.; Khotkevich, V. V.; Morozov, S. V.; Geim, A. K., Proc. Natl. Acad. Sci. U. S. A. 2005, 102, 1045110453.

(3) (a) Li, L. K.; Yu, Y. J.; Ye, G. J.; Ge, Q. Q.; Ou, X. D.; Wu, H.; Feng, D. L.; Chen, X. H.; Zhang, Y. B., Nat. Nanotechnol. 2014, 9 , 372-377. (b) Liu, H.; Neal, A. T.; Zhu, Z.; Luo, Z.; Xu, X. F.;
Tomanek, D.; Ye, P. D., ACS Nano 2014, 8, 4033-4041.

(4) Wang, C. L.; Dong, H. L.; Hu, W. P.; Liu, Y. Q.; Zhu, D. B., Chem. Rev. 2012, 112, 2208-2267.

(5) (a) Arias, A. C.; MacKenzie, J. D.; McCulloch, I.; Rivnay, J.; Salleo, A., Chem. Rev. 2010, 110, 3-24. (b) Reyes-Martinez, M. A.; Crosby, A. J.; Briseno, A. L., Nat. Commun. 2015, 6, 6948.

(6) Jiang, L.; Gao, J.; Wang, E.; Li, H.; Wang, Z.; Hu, W.; Jiang, L., Adv. Mater. 2008, 20, 2735-2740.

(7) Tang, Q.; Li, L.; Song, Y.; Liu, Y.; Li, H.; Xu, W.; Liu, Y.; Hu, W.; Zhu, D., Adv. Mater. 2007, 19, 2624-2628.

(8) Shim, H.; Kumar, A.; Cho, H.; Yang, D.; Palai, A. K.; Pyo, S., ACS Appl. Mater. Interfaces 2014, 6, 17804-17814.

(9) Min, S. Y.; Kim, T. S.; Kim, B. J.; Cho, H.; Noh, Y. Y.; Yang, H.; Cho, J. H.; Lee, T. W., Nat. Commun. 2013, 4, 1773.

(10) Haddock, J. N.; Zhang, X. H.; Zheng, S. J.; Zhang, Q.; Marder, S. R.; Kippelen, B., Org. Eelectron. 2006, 7, 45-54.

(11) Luo, C.; Kyaw, A. K. K.; Perez, L. A.; Patel, S.; Wang, M.; Grimm, B.; Bazan, G. C.; Kramer, E. J.; Heeger, A. J., Nano Lett. 2014, 14, 2764-2771.

(12) Rekab, W.; Stoeckel, M. A.; El Gemayel, M.; Gobbi, M.; Orgiu, E.; Samori, P., ACS Appl. Mater. Interfaces 2016, 8, 9829-9838.

(13) (a) Zhang, L.; Zhong, X.; Pavlica, E.; Li, S.; Klekachev, A.; Bratina, G.; Ebbesen, T. W.; Orgiu, E.; Samori, P., Nat. Nanotechnol. 2016, 11, 900-906. (b) Zhang, L.; Pavlica, E.; Zhong, X.; Liscio, F.; Li, S.; Bratina, G.; Orgiu, E.; Samori, P., Adv. Mater. 2017, 29, 1605760 .

(14) (a) Wang, H.; Cheng, C.; Zhang, L.; Liu, H.; Zhao, Y.; Guo, Y.; Hu, W.; Yu, G.; Liu, Y., Adv. Mater. 2014, 26, 4683-4689. (b) El Gemayel, M.; Treier, M.; Musumeci, C.; Li, C.; Mullen, K.; Samori, P., J. Am. Chem. Soc. 2012, 134, 2429-2433.

(15) Baeg, K. J.; Binda, M.; Natali, D.; Caironi, M.; Noh, Y. Y., Adv. Mater. 2013, 25, 4267-4295.

(16) Noh, Y. Y.; Ghim, J.; Kang, S. J.; Baeg, K. J.; Kim, D. Y.; Yase, K., J. Appl. Phys. 2006, 100, 094501.

(17) (a) Zhang, J.; Xu, W.; Sheng, P.; Zhao, G. Y.; Zhu, D. B., Acc. Chem. Res. 2017, 50, 1654-1662. (b) Nakano, K.; Tajima, K., Adv. Mater. 2017, 29, 1603269. (c) Li, H.; Fan, C.; Fu, W.; Xin, H. L.; Chen, H., Angew. Chem. Int. Ed. Engl. 2015, 54, 956-960. (d) Zhang, Y. J.; Dong, H. L.; Tang, Q. X.; Ferdous, S.; Liu, F.; Mannsfeld, S. C. B.; Hu, W. P.; Briseno, A. L., J. Am. Chem. Soc. 2010, 132, 1158011584.

(18) Wang, S.; Zhang, L. H.; Zhang, Z. Y.; Ding, L.; Zeng, Q. S.; Wang, Z. X.; Liang, X. L.; Gao, M.; Shen, J.; Xu, H. L.; Chen, Q.; Cui, R. L.; Li, Y.; Peng, L. M., J. Phys. Chem. C 2009, 113, 68916893.

(19) Li, Y. F., Acc. Chem. Res. 2012, 45, 723-733. 